\title{
The influence of allopurinol and post-conditioning on lung injuries induced by lower-limb ischemia and reperfusion in Wistar rats ${ }^{1}$
}

Hugo Genki Kagawa Akahane', Ricardo Zanetti Gomes", Katia Sabrina Paludo", Filipe Linhares"', Luana Lopes ${ }^{\prime \prime \prime}$

'Graduate student, School of Medicine, Universidade Estadual de Ponta Grossa (UEPG), Brazil. Scientific and intellectual content of the study, acquisition of data, technical procedures, manuscript preparation.

"PhD, Head, Department of Medicine, UEPG, Ponta Grossa-PR, Brazil. Scientific, intellectual, conception and design of the study; interpretation of data; critical revision.

"'Graduate student, School of Medicine, UEPG, Ponta Grossa-PR, Brazil. Technical procedures, acquisition of data.

\section{Abstract}

Purpose: To analyze the effects of allopurinol and of post-conditioning on lung injuries induced by lower-limb ischemia and reperfusion.

Methods: Thirty rats were used. They were divided in 5 groups: (1) group A: abdominal aortic dissection only, (2) group B: ischemia and reperfusion, (3) group C: administered allopurinol $(100 \mathrm{mg} / \mathrm{Kg}$ ) a few hours before procedure, (4) group D: post-conditioned and (5) group E: administered allopurinol and post-conditioned. With the exception of group A, all groups were submitted to infrarenal aortic ischemia for 2 hours, and reperfusion for 72 hours. After euthanasia, lungs were removed for histological analysis. They were graded under two scores: pulmonary injury (neutrophil infiltration, interstitial edema, vascular congestion, and destruction of lung architecture) and lymphocytic score (neutrophil infiltration, lymphoid aggregate and secondary follicle).

Results: On the pulmonary injury score, the degree of injury was smaller than in groups $D$ and $E$, when compared to group $B, p<0.05$. Group $C$ did not obtain the same result $(p>0,05)$. On the lymphocytic score, there was no statistic difference among groups, $p>0.05$.

Conclusion: Both post-conditioning and the combination of allopurinol and post-conditioning were effective in remote lung protection induced by lower-limbs I/R. When used in isolation, allopurinol showed no protective effect.

Key words: Allopurinol. Ischemic Postconditioning. Reperfusion. Reperfusion Injury. Lung 


\section{Introduction}

Ischemia is defined as the reduction of blood supply to a tissue, with ensuing diminution of oxygen and nutrient supply ${ }^{1,2}$. Contradictorily, when blood flow is reestablished, the injury is exacerbated, proceeding at a high pace, and causing injury by ischemia and reperfusion $(\mathrm{I} / \mathrm{R})^{1-3}$.

During ischemic process, the cell utilizes anaerobic metabolism as energetic resource, thus creating an environment rich in lactic acid ${ }^{2,3}$. Besides the adenosine triphosphate (ATP) depletion into adenosine diphosphate, adenosine monophosphate and adenosine ${ }^{2,3}$. The latter is diffused to extracellular environment, where it is degraded into inosine and hypoxanthine ${ }^{2,3}$. During the hypoxia period, the accumulation of intracellular calcium activates intracellular proteases, transforming xanthine dehydrogenase into xanthine oxygenase $\mathrm{e}^{2-4}$. In the reperfusion, while oxygen is active, xanthine oxidase metabolizes hypoxanthine in reactive oxygen species (ROS), which are: anion superoxide, hydrogen peroxide and the radical hydroxila ${ }^{3,5}$. Which can a cause both local and systemic injuries, especially in lipid and protein compounds, and in the cell's nucleic acids ${ }^{3,6}$.

Allopurinol has been amply used as antioxidant method in I/R experimental models, because it presents a structure analogous to that of hypoxanthine, as it is a competitive inhibitor of xanthine oxidase, with consequent reduction in ROS production, particularly the superoxide radical ${ }^{7-9}$.

Ischemic Post-conditioning (IPostC) consists in short reperfusion cycles interpolated by short ischemic cycles, immediately after the ischemia stage and before permanent reperfusion ${ }^{11}$. The mechanisms involved in IPostC are not yet entirely clear. It is hypothesized that it diminishes ROS production, due to the slow liberation of oxygen to the tissue, counterpoising its productive peak in single reperfusion, with prevention of injuries by $\mathrm{I} / \mathrm{R}^{10-12}$.

This study intends to analyze the effects of allopurinol and IPostC on lower-limb lung injuries secondary to $\mathrm{I} / \mathrm{R}$ in Wistar rats.

\section{Methods}

All procedures were evaluated and approved by the university's Animal Ethics Committee, and registered under number 24/2014.

This paper is a controlled randomized experimental study. It used 30 Wistar rats, all female, approximately 3 months old, with weight ranging from 250 to $300 \mathrm{~g}$, from Universidade Estadual de Ponta Grossa and Pontifícia Universidade Católica. The animals were maintained in the UEPG vivarium during the experiment, with enough food and water. The surgical procedures were conducted at the Operation Technology and Experimental Surgery or Multidisciplinary Laboratory, UEPG.

The animals were randomly divided in five groups:

Group A: SHAM group, underwent infrarenal aortic dissection only;

Group B: control group, underwent twohour ischemia, followed by single reperfusion for 72 hours;

Group C: were administered allopurinol. Underwent two-hour ischemia and single reperfusion;

Group D: underwent two-hour ischemia, followed by IPostC before final reperfusion;

Group E: combined therapy. Were administered a single dose of allopurinol. Underwent two-hour ischemia and IPostC before final reperfusion.

\section{Anesthesia procedures}

The animals' anesthesia induction was conducted by association of a $75 \mathrm{mg} /$ 
$\mathrm{kg}$ dose of ketamine with a $10 \mathrm{mg} / \mathrm{kg}$ dose of xylazine, both administered intraperitoneally. Anesthesia was maintained during the procedure by using half of the initial dosage, administered intramuscularly (upper limb), in order to maintain the anesthesia plan.

\section{Surgical procedure}

After confirmation that the animals were anesthetized, by corneal and inter-digital reflex, they were fixed in dorsal decubitus, submitted to broad abdominal epillation, followed by antisepsis with alcohol iodate. A laparotomy by median xypho-pubic incision was then performed, with aperture in the planes of the abdominal wall and exposition of the posterior parietal peritoneum, and separation of the inferior vena cava from the infrarenal aorta. Groups C and E were probegavaged with a $100 \mathrm{mg} / \mathrm{Kg}$ dose of allopurinol one hour before the procedure. Groups B, C, D and $E$ had the infrarenal aorta clamped for two hours, which was doubly surrounded by elastic bands. Group A underwent dissection of the infrarenal aorta only, and was not submitted to ischemia. In the meantime, the intestinal loops were kept moist with gaze immersed in a heated isotonic saline solution, to avoid loss of liquid by evaporation. Groups D and E underwent IPostC, with three ischemic episodes and three reperfusions, with duration of two minutes each (ischemic post-conditioning). After ischemia, the tapes were removed and the presence of distal pulse verified, followed by the closure of the abdominal wall in two planes, muscular and skin, by running sutures. The animals were then sent to the vivarium, and maintained with enough food and water.

After 72 hours, the animals were anesthetized with ketamine and xylazine, doses of, respectively, 75 and $10 \mathrm{mg} / \mathrm{kg}$. The sacrifice was conducted by exsanguination, by punction of the carotid artery, with a help of an intravenous device, a plastic catheter on a needle (abbocath). After the death of the animal, the lungs were removed for histologic analysis.

\section{Histologic analysis}

For histologic analysis, the inferior lobe of the left lungs of all animals was collected, 72 hours after the procedure. The samples were fixed in ALFAC (85\% to $80 \%$ ethanol, $5 \%$ glacial acetic acid and $10 \%$ formaldehyde) for sixteen hours. The tissues were next dehydrated in consecutive ethanol baths, and diaphanized with xylene. They were included in paraffin, cut with a microtome in sections of 5 micrometres each, and dyed with haematoxylin and eosin.

Two scales were set up for the histological analysis: pulmonary injure score and lymphocytic score. The pulmonary injury score consisted of the following parameters: (1) neutrophil infiltration, (2) interstitial edema, (3) vascular congestion, and (4) destruction of lung architecture. The lymphocytic score consisted of: (1) neutrophil infiltration, (2) lymphoid aggregate and (3) presence of secondary follicles.

In both scores, the parameters were graded according to histological scale. Zero was considered normal, 1 discreet, 2 light, 3 moderate and 4 heavy.

The score was established based on the total sum of the values of the histological scales obtained for each parameter.

All samples were tested for normality using the Shapiro-Wilk Test. The Kruskal-Wallis test was used for statistic comparison, with values $p<0.05$ being considered relevant.

\section{Results}

Upon analysis, the pulmonary injury score obtained the following medians: Group A, 5; Group B, 9; Group C, 8.5; Group D, 8; Group E, 6. These results are seen in Table 1. 
Table 1 - Histological analysis results (pulmonary injury score).

\begin{tabular}{llllll} 
Rats & \multicolumn{6}{l}{ Pulmonary Injury Score } \\
\hline \multirow{5}{*}{} & Group & Group & Group & Group & Group \\
1 & A & B & C & D & E \\
2 & 7 & 9 & 7 & 6 & 6 \\
3 & 5 & 9 & 10 & 5 & 5 \\
4 & 5 & 9 & 9 & 8 & 7 \\
5 & 5 & 10 & 8 & 8 & 7 \\
6 & 6 & 9 & 9 & 8 & 6 \\
7 & 5 & 9 & 8 & 9 & - \\
Median & 5 & - & - & - & - \\
\hline & 5.0 & 9.0 & 8.5 & 8.0 & 6.0 \\
\hline
\end{tabular}

Comparison of data for the pulmonary injury score from Group B (9) and Groups D and $E$ ( 8 and 6 , respectively) obtained $p<0.05$, whereas Group C (8.5) did not obtain the same result $(p>0.05)$. It was also observed that Groups B, C and D obtained a higher degree of injury, when compared to Group $A(5 ; p<0.05)$; the same cannot be observed for Group $E$ in Figure 1.

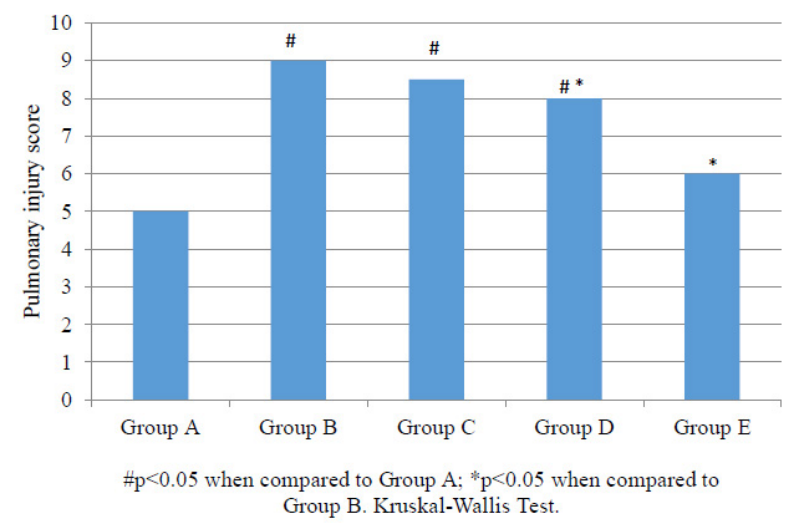

Figure 1 - Comparison for pulmonary injury score among groups.

The four variables were observed individually: neutrophil infiltration, interstitial edema, vascular congestion, and destruction of lung architecture:
The degree of neutrophil infiltration for groups $A, C, D$ and $E$ were the same (median = 1), whereas Group B obtained 2, $p<0.05$ (Figure 2). There was also a difference in the degree of interstitial edema in groups $A, D$ and $E$ (all of which obtained median $=1$ ) when compared to Group B $(2 ; p<0.05)$ in Figure 3. However, no statistic difference was observed for the parameters vascular congestion and destruction of lung architecture, when compared to Group B.

The degrees of interstitial edema (Figure 3) and architecture destruction were high for both Groups B ( 2 and 3, respectively) and $C$ ( 2 and 3 ), when compared to Group A (1 e $2 ; p<0.05)$. Groups B, C, D and E obtained a significant raise as to their degrees of vascular congestion (median $=2$ ), when compared to Group A $(1 ; p<0.05)$.

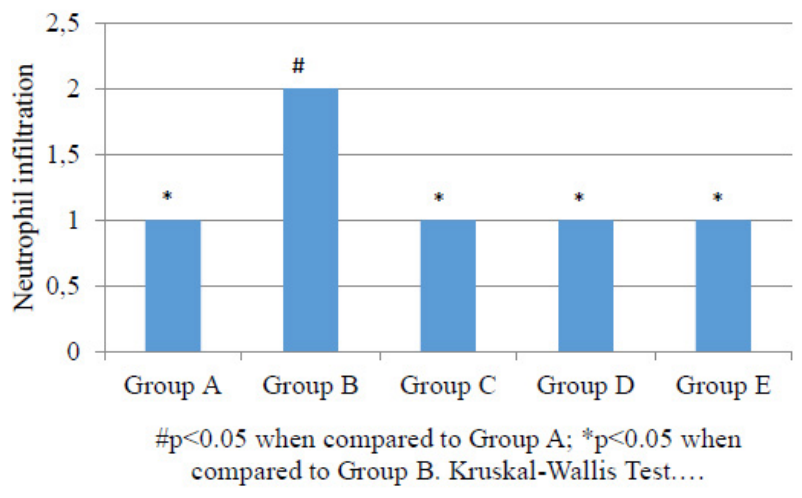

Figure $\mathbf{2}$ - Comparison for neutrophil infiltration among groups

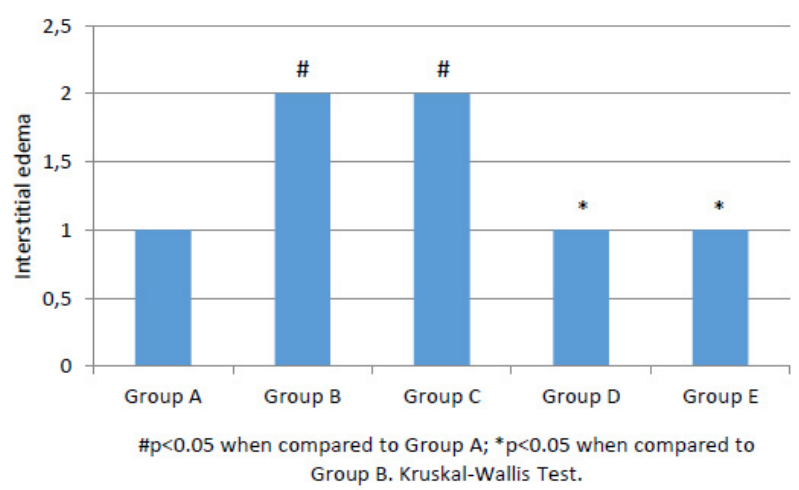

Figure 3-Comparison for interstitial edema among groups. 
In the lymphocytic score (Table 2), the medians obtained for each group were the following: Group $A=3$, Group B = 5; Group C = 3.5; Group $D=3.5$ and Group $E=4$. There was not statistic difference among groups, $p>0.05$. Upon individual analysis, the lymphocytic infiltration degree was higher for groups B, Cand E (2 for each group), when compared to group A $(1 ; p<0.05)$. Comparison for the parameters lymphoid aggregates and secondary follicles obtained no statistic difference.

Table 2 - Histological analysis results (lymphocytic score).

\begin{tabular}{llllll} 
Rats & \multicolumn{6}{l}{ Lymphocytic score } \\
\hline & Group & Group & Group & Group & Group \\
& A & B & C & D & E \\
1 & 3 & 5 & 3 & 3 & 7 \\
2 & 3 & 8 & 2 & 3 & 3 \\
3 & 1 & 8 & 3 & 6 & 4 \\
4 & 6 & 4 & 5 & 5 & 6 \\
5 & 3 & 5 & 4 & 4 & 4 \\
6 & 3 & 3 & 4 & 2 & - \\
7 & 1 & - & - & - & - \\
Median & 3.0 & 5.0 & 3.5 & 3.5 & 4.0 \\
\hline
\end{tabular}

\section{- Discussion}

Despite the fact that reperfusion is an essential phenomenon, fundamental for tissue vitality and recovery, during the I/R process, the opposite is verified ${ }^{1-3}$. There is an increase in local injury, necrosis and rhabdomyolysis, as well as of their systemic repercussions. The metabolics and pro-inflammatory cytokines originating from muscular injury may induce Systemic Inflammatory Response Syndrome, and may affect distant organs, such as kidneys, lung and gastrointestinal tract - multiple organ dysfunction ${ }^{13}$. Among these organs, the lung was the one analyzed in the present study.

Lungs are among the organs most susceptible to multiple organ dysfunctions.
It may be remotely injured by reperfusion in organs such as intestines, kidneys, liver and skeletal muscle ${ }^{14}$, resulting in noncardiogenic pulmonary edema ${ }^{15}$. Among the mediators involved in $\mathrm{I} / \mathrm{R}$ injury, there were identified: ROS, lipid mediators such as thromboxane, neutrophils and endothelial adhesion molecules, inflammatory cytokines, as tumor necrosis factor alpha and interleukins 1, 6 and 8, and activated neutrophils, with consequent raise in vascular and alveolarmembrane permeability ${ }^{14}$. For this reason, the parameters analyzed were the degrees of neutrophil infiltration, interstitial edema, vascular congestion, and destruction of lung architecture. Given this physiopathology, the following are used as therapeutic measures for reperfusion injury: antioxidants, lipid mediator blockers, leukocyte-endothelial cell interaction-inhibitors, or substances favoring post-ischemic blood flow ${ }^{5,16}$.

Experimental studies in animals have demonstrated the efficiency of antioxidant therapy in attenuating or preventing I/R injury; among the antioxidants tested, we may cite: melatonin, Vitamin E, superoxide dismutase, catalase, mannitol, chelated iron complexes, calcium-channel blockers, $\mathrm{N}$-acetylcysteine and allopurinol, the latter being the object of the present study ${ }^{2,5}$. By means of lipid peroxidation, ROS damage cell membranes, resulting in the production of malondialdehyde, a lipid peroxidation marker ${ }^{5}$.

Using $\mathrm{N}$-acetylcysteine as antioxidant and pentoxifylline as a non-specific phosphodiesterase inhibitor, Takhtfooladi et $a .^{16}$ demonstrated that both presented protective results in remote lung injury to skeletal muscle I/R, attributed to the inhibition in ROS production. However, their combined use has not proven beneficial ${ }^{16}$. The study also reported an association between medium lung injury by skeletal muscle I/R-induced oxidation reactions when observing a significant raise 
on malondialdehyde levels, and the disarray of lung histology after remote $1 / \mathrm{R}^{16}$. Give the protective effects of antioxidants, a series of analyses on the effects of allopurinol in reducing lower-limb I/R remote lung injury was conducted.

Allopurinol presents a structure analogous to hypoxanthine, acting as xanthine oxidase inhibitor, and as ROS-production neutralizer. Additionally, it minimizes neutrophil activation and inflammation, and diminishes xanthine oxidase circulation, which are related to endothelial dysfunction and remote organ damage ${ }^{5}$. In the present study, Group C (which was administered allopurinol only) did not demonstrate less remote injury on lung histology when compared to Group B (ischemia and single reperfusion), especially relating the degrees of interstitial edema and destruction of lung architecture. This is corroborated by Gomes et al. ${ }^{17}$, which analyses indirect intestinal injury by lower-limb $\mathrm{I} / \mathrm{R}$, by bacterial translocation; this study shows that neither allopurinol in isolation nor associated with gentamicin were able to reduce bacterial translocation.

On the other hand, in the literature there are several studies demonstrating the beneficial effects of allopurinol as antioxidant. Gomes et al. ${ }^{18}$ demonstrates I/R-induced inflammation reduction, when comparing the efficiency of allopurinol in reducing serial concentration of tumor necrosis factors, of the I/R group, with groups submitted to I/R and treated with allopurinol. Allopurinol also displayed significant advantages in reducing injury by reperfusion in the kidneys of rats, which was demonstrated by Keel et al. ${ }^{19}$, by the utilization of 8-isoprostane as oxidative stress marker in the evaluation of injury by I/R; Zhou et al. ${ }^{20}$ evidenced improvement in kidney function and histology. In a small-intestine I/R model, histological analysis demonstrated allopurinol, administered before ischemia and reperfusion, to display moderate intestinal mucosa damage, and smaller production of malondialdehyde, when compared to the control group ${ }^{21}$. In upper-limp experiments, associated to I/R for six hours, both the administration of allopurinol and streptokinase have demonstrated a raise in the viability of skeletal muscle from $50 \%$ to 64\% (streptokinase) and 70\% (allopurinol) ${ }^{22}$.

With the data obtained, comparing group D (submitted to IPostC) to Group B (submitted to ischemia and single reperfusion), there was an improvement in parameters of pulmonary histology, $p<0.05$. Studies show the efficiency of IPostC as cardioprotector in cardiac revascularization surgery, with smaller muscle injury; this concept was first described in 2003 by Zhao et al. ${ }^{23}$, which demonstrates its higher efficiency in relation to preconditioning, in myocardial revascularization surgery. Recently, this concept was expanded by experimental lower-limb I/R models, by infrarenal aorta clamping, simulating abdominal aorta surgery ${ }^{24}$. Gyurkovics et al. ${ }^{24}$ were the first to describe this study, in which it is demonstrated the efficiency of IPostC in minimazing systemic damage and multipleorgan complications resulting from lower-limb I/R. IPostC was efficient in the reduction of lung and kidney remote injury, tumor necrosis factor and $\mathrm{ROS}^{24}$. The histological analyses of the I/R group showed areas of collapsed alveoli and thickening of alveolar walls, whereas in the IPostC group the alterations were minimal, corroborating the protective effect of IPostC in the present study ${ }^{24}$.

As for the comparatively smaller degree of lung injury in group $D$ in relation to Group B, Garbaisz et al. ${ }^{25}$ obtained the same results; in their studies, they demonstrated reduction in ROS and in tumor necrosis factor, when comparing IPostC with groups submitted to infrarenal aorta $\mathrm{I} / \mathrm{R}^{25}$. In histological analysis, the IPostC group showed fewer areas of atelectasis, bigger alveolar space, and less thickening of the 
alveolar wall ${ }^{25}$. Lung tissue myeloperoxidase activity was smaller in the IPostC group when compared to the I/R group, demonstrating less polymorphonuclear sequestration and activation in the lung parenchyma ${ }^{25}$. Garbaisz et al..$^{25}$ have thus demonstrated that IPostC was capable of reducing inflammatory response and lung injury by infrarenal aorta clamping ${ }^{25}$.

Group E (administered allopurinol and (PostC) also obtained positive results in reduction of lung injury, in relation to Group $B, p<0.05$. However, no data was found in the literature reporting therapeutic association of allopurinol and IPostC. It was observed that Group E obtained a smaller median when compared to Group D (6 and 8, respectively), despite there being no statistic difference ( $p>0.05)$.

Countering the literature, the histological analyses conducted in the present study showed a higher degree of lymphocytic infiltration when compared to neutrophil, as well as areas of lymphoid tissue hyperplasia. This may be explained by endotoxemia ${ }^{26}$, by lipopolysaccharides resulting from break of the intestinal barrier by remote injury. In a study on the induction of acute respiratory anguish syndrome by intraperitoneal administration of lipopolysaccharides, Bánfi et al. ${ }^{26}$ reported predominance of lymphoid tissue hyperplasia associated to the bronchus, with a smaller degree of characteristics of the acute phase (edema, alveolar hyperemia, hemorrhaging, type II pneumocytes alteration, alveolar macrophage accumulation, and development of hyalinemembrane). Lauet al. ${ }^{27}$ demonstrated that the simple manipulation of the intestinal loops culminates in endotoxemia, and breaks the intestinal barier ${ }^{28}$. The relation was also investigated with a 72-hour reperfusion time, with accelerated metabolic rate, given that, in other studies, reperfusion time was 2 hours ${ }^{29}, 4$ hours $^{24,25}$ e 24 hours $^{14,15}$; the hypothesis raised was that there had already been a resolution to the acute phase of neutrophil infiltration, with predominance of monomorphonuclear cells ${ }^{30}$.

Abnormal lung histology data for Group A are to be questioned, due to the great stress the animals were submitted to by laparotomy by xypho-pubic incision, alongside with manipulation of the intestinal loops, thus favoring endotoxemia ${ }^{27,28}$.

\section{- Conclusion}

Both post-conditioning and the combination of post-conditioning and allopurinol were effective in causing remote lung protection induced by lower-limb I/R. When isolated, allopurinol demonstrated no protective effect.

\section{References}

1. Reimer KA, Jennings $R B$, Tattum $A H$. Pathobiology of acute myocardial ischemia: metabolic functional and ultra structural studies. Am J Cardiol. 1983;52:72A-81. doi: 10.1016/0002-9149(83)90180-7.

2. Silveira $M$, Yoshida WB. Isquemia e reperfusão em músculo esquelético: mecanismos de lesão e perspectivas de tratamento. J Vasc Bras. 2004;3(4):367-78.

3. Sanada S, Komuro I, Kitakaze M. Pathophysiology of myocardial reperfusion injury: preconditioning, postconditioning, and translational aspects of protective measures. Am J Physiol Heart Circ Physiol. 2011;301(5):1723-41. doi: 10.1152/ ajpheart.00553.2011.

4. Ferreira ALA, Matsubara LS. Radicais livres: conceitos, doenças relacionadas, sistema de defesa e estresse oxidativo. Rev Assoc Méd Bras. 1997;43(1):61-doi: 10.1590/S010442301997000100014.

5. Pinheiro BV, Holanda MA, Araújo FG, Romaldini, $H$. Lesão pulmonar de reperfusão. J Pneumol. 1999;25(2):124-36. doi: 10.1590/S0102-35861999000200010.

6. Angela PC, Miguel AMCJ, Susi L, Emilio F, Manuel JS, Djalma JF. The role of $\mathrm{N}$-acetylcysteine in the lung remote injury after 
hepatic ischemia and reperfusion in rabbits. Acta Cir Bras. 2012,27(1):49-55. doi: 10.1590/S0102-86502012000100009.

7. Rhoden EL, Mauri M, Petteffi L, Dacanal F, Pilla M, Belló-Klein A, Cláudio T, Elvino $B$, Rhoden CR. Efeitos do alopurinol sobre a lipoperoxidação de membranas celulares renais na síndrome da isquemia e reperfusão: estudo experimental em ratos. Acta Cir Bras. 1998;13(2):73-9. doi: 10.1590/ S0102-86501998000200002.

8. Cunha MS, Silva JCF, Nakamoto $H$, Simão DT, Ferreira MC. Efeito do alopurinol e terapia com oxigênio hiperbárico em modelo de reimplante de membro após isquemia quente em ratos. Rev Col Bras Cir. 2005;32(2):64-8. doi: 10.1590/S010069912005000200004.

9. Rhoden EL, Mauri M, Rhoden CR, Leal MLM, Sabedotti M, Lucas ML, Pereira-Lima L. Taxa de mortalidade em ratos submetidos à isquemia e reperfusão hepática, tratados ou não com alopurinol. Acta Cir Bras. 1999;14(4):166-70.

10.Zhao ZQ, Corvera JS, Halkos ME, Kerendi F, Wang NP, Guyton RA, Vinten-Johansen J. Inhibition of myocardial injury by ischemic postconditioning during reperfusion: comparison with ischemic preconditioning. Am J Physiol Heart Circ Physiol. 2003;285(2):579-88. doi: 10.1152/ ajpheart.01064.2002.

11. Cinel I, Avlan D, Cinel L, Polat G, Atici S, Mavioglu I, Serinol H, Aksoyek S, Oral $U$. Ischemic preconditioning reduces intestinal epithelial apoptosis in rats. Shock. 2003;19(6):588-92. doi: 10.1097/01. shk.0000055817.40894.84.

12. Ke JJ, Yu FX, Rao Y, Wang YL. Adenosine postconditioning protects against myocardial ischemia-reperfusion injury though modulate production of TNF-a and prevents activation of transcription factor NF-kB. Mol Biol Rep. 2011;38(1):531-8. doi: 10.1007/s11033-010-0137-8.

13.Garbaisz D, Turoczi Z, Aranyi P, Fulop A, Rosero O, Hermesz E, Ferencz A, Lotz $G$, Harsanyi L, Szijarto A. Attenuation of skeletal muscle and renal injury to the lower limb following ischemia-reperfusion using mPTP inhibitor NIM-811. PLoS One. 2014 Jun 26;9(6):e101067. doi: 10.1371/journal. pone.0101067.
14.Takhtfooladi MA, Jahanshahi A, Sotoudeh A, Jahanshahi G, Takhtfooladi HA, Aslani K. Effect of tramadol on lung injury induced by skeletal muscle ischemia-reperfusion: an experimental study. J Bras Pneumol. 2013;39(4):434-9. doi: 10.1590/S180637132013000400006.

15.Lv X, Wang ZM, Huang SD, Song SH, Wu FX, YuWF. Emulsified isoflurane preconditioning reduces lung injury induced by hepatic ischemia/reperfusion in rats. Int J Med Sci. 2011;8(5):353-61. doi: 10.7150/ijms.8.353.

16. Takhtfooladi HA, Hesaraki S, Razmara F, Takhtfooladi MA, Hajizadeh $\mathrm{H}$. Efeitos da $\mathrm{N}$-acetilcisteína e pentoxifilina na lesão pulmonar remota em um modelo de lesão de isquemia/reperfusão de membro posterior em ratos. J Bras Pneumol. 2016;42(1):9-14. doi: 10.1590/S1806-37562016000000183.

17.Gomes RZ, Bittencourt JM, Galdino DT, Campanerutti GA. Influência do alopurinol e da gentamicina nos efeitos da isquemia e reperfusão de membros caudais em ratos Wistar. Rev Bras Med Interna. 2014;1(1):3642. doi: 10.15743/rbmi.2014.0005.

18.Gomes RZ, Romanek GMM, Przybycien M, Amaral DC, Akahane HGKA. Evaluation of the effect of allopurinol as a protective factor in post ischemia and reperfusion inflammation in Wistar rats. Acta Cir Bras. 2016;31(2):126-32. doi: 10.1590/S0102865020160020000007.

19.Keel CE, Wang Z, Colli J, Grossman L, Majid $D$, Lee BR. Protective effects of reducing renal ischemia-reperfusion injury during renal hilar clamping: use of allopurinol as a nephroprotective agent. Urology. 2013 Jan;81(1):210.e5-10. doi: 10.1016/j. urology.2012.08.016.

20.Zhou J, Qiu T, Zhang L, Chen Z, Wang Z, $\mathrm{Ma} X, \mathrm{Li}$ D. Allopurinol preconditioning attenuates renal ischemia/reperfusion injury by inhibiting HMGB1 expression in a rat model. Acta Cir Bras. 2016;31(3):176-82. doi: 10.1590/S0102-865020160030000005.

21.Sapalidis K, Papavramidis TS, Gialamas E, Deligiannidis N, Tzioufa V, Papavramidis S. The role of allopurinol's timing in the ischemia reperfusion injury of small intestine. J Emerg Trauma Shock. 2013;6(3):203-8. doi: 10.4103/0974-2700.115346.

22.Cunha MS, Silva JCF, Nakamoto HA, Ferreira MC. Study of warm ischemia followed by 
reperfusion on a lower limb model in rats: effect of allopurinol and streptokinase. Clinics. 2005;60(3):213-20. doi: 10.1590/ S1807-59322005000300006.

23.Zhao ZQ, Corvera JS, Halkos ME, Kerendi F, Wang NP, Guyton RA, Vinten-Johansen J. Inhibition of myocardial injury by ischemic postconditioning during reperfusion: comparison with ischemic preconditioning. Am J Physiol Heart Circ Physiol. 2003;285(2):579-88. doi: 10.1152/ ajpheart.01064.2002.

24.Gyurkovics E, Aranyi P, Stangl R, Onody P, Ferreira G, Lotz G, Kupcsulik P, Szijarto A. Postconditioning of the lower limb protection against the reperfusion syndrome. J Surg Res. 2011;169(1):139-47. doi: $10.1016 / j$.jss.2009.10.014.

25.Garbaisz D, Turoczi Z, Fulop A, Rosero $O$, Aranyi $P$, Onody P. Therapeutic option for managing lung injury induced by infrarenal aortic cross-clamping. J Surg Res. 2013;185(1):469-76. doi: 10.1016/j. jss.2013.05.022.

26.Bánfi A, Tiszlavicz L, Székely E, Peták F, Tóth-Szuki V, Baráti L. Development of bronchus-associated lymphoid tissue hyperplasia following lipopolysaccharide- induced lung inflammation in rats. Exp Lung Res. 2009;35(3):186-97 doi: $10.1080 / 01902140802495862$.

27.Lau LL, Halliday MI, Lee1 B, Hannon1 RJ, Gardiner KR, Soong1 CV. Intestinal manipulation duringelectiveaorticaneurysm surgery leads to portal endotoxaemia and mucosal barrier dysfunction. Eur J Vasc Endovasc Surg. 2000;19(6):619-24. doi: 10.1053/ejvs.2000.1063.

28. Norwood MGA, Bown MJ, Sayers RD. Ischaemia-reperfusion injury and regional inflammatory responses in abdominal aortic aneurysm repair. Eur J Vasc Endovasc Surg. 2004;28(3):234-45. doi: 10.1016/j. ejvs.2004.03.026.

29. Koksela O, Yildirima C, Cinelb L, Tamerc L, Ozdulgera A, Basturkb M, Degirmenci $U$, Kanik A, Cinel I. Inhibition of poly(ADPribose) polymerase attenuates lung tissue damage afterhind limb ischemia-reperfusion in rats. Pharmacol Res. 2005;51(5):45362. doi: 10.1016/j.phrs.2004.11.007 PMid:15749460.

30. Matthay MA, Zemans RL. The acute respiratory distress syndrome: pathogenesis and treatment. Pathology. 2011;6:147-63. PMID: 20936936.

\section{Correspondence:}

Ricardo Zanetti Gomes

Departamento de Medicina, Universidade

Estadual de Ponta Grossa

Avenida General Carlos Cavalcanti, 4748

84030-900 Ponta Grossa - PR Brasil

Tel.: (55 42)3220-3000 / 99972-0704

zanetticons@uol.com.br

Received: May 17, 2017

Review: July 18, 2017

Accepted: Aug 18, 2017
Conflict of interest: none

Financial source: none

\footnotetext{
${ }^{1}$ Research performed at Experimental Surgery and Operation Technique Laboratory, Department of Medicine, Universidade Estadual de Ponta Grossa (UEPG), Brazil.
} 